\title{
Method development and validation of nitrofuran metabolites in shrimp by liquid chromatographic mass spectrometric system
}

\begin{abstract}
Liquid Chromatographic Mass Spectrometric System (LCMSMS) method was developed and validated in shrimp matrix for determination of nitrofuran metabolites in the laboratory of fish inspection and quality control, Department of Fisheries, Matshya Bhaban, Dhaka, Bangladesh. This confirmatory method was used for testing samples for residues of the metabolites of the following nitrofuran compounds, furazolidone (3-amino-2-oxazolidinone, AOZ); furaltadone (5-methyl-morpholino-3-amino-2-oxazolidinone, AMOZ); nitrofurazone (semicarbazide, SEM) and nitrofurantoin (1-aminohydantoin, AHD) in shrimp. The data were generated (3 levels and seven replicates per level) on each of three days for shrimp. The mean recoveries from the tissues were 88-110\%, the decision limits (CCUั) were 0.12-0.23 ppb and the detection capabilities (CCŬ) $0.21-0.38 \mathrm{ppb}$. CCŬ and ССБ were calculated using the procedure set out in ISO Guide 11843. Nevertheless, CCǓvalue for all nitrofuran metabolites can be investigated and meet the specified easily met the specified EU MRPL of 1 हg kg-1 ranged from $0.12-0.23 \varepsilon \mathrm{g} \mathrm{kg}-1$ and suitable for routine quality control operations.
\end{abstract}

Keyword: Decision limit; Detection capability; Method validation; Nitrofuran; Shrimp 